Copyright by the Acoustical Society of America. Esteve, S. J., \& Johnson, M. E. (2002). Reduction of sound transmission into a circular cylindrical shell using distributed vibration absorbers and Helmholtz resonators. Journal of the Acoustical Society of America, 112(6), 2840-2848. doi: 10.1121/1.1514933

\title{
Reduction of sound transmission into a circular cylindrical shell using distributed vibration absorbers and Helmholtz resonators
}

\author{
Simon J. Estève ${ }^{\text {a) }}$ and Marty E. Johnson \\ Vibration and Acoustics Laboratories, Department of Mechanical Engineering, \\ Virginia Polytechnic Institute and State University, Blacksburg, Virginia 24061
}

(Received 4 March 2002; revised 29 July 2002; accepted 22 August 2002)

\begin{abstract}
A modal expansion method is used to model a cylindrical enclosure excited by an external plane wave. A set of distributed vibration absorbers (DVAs) and Helmholtz resonators (HRs) are applied to the structure to control the interior acoustic levels. Using an impedance matching method, the structure, the acoustic cavity, and the noise reduction devices are fully coupled to yield an analytical formulation of the structural kinetic energy and acoustic potential energy of a treated cylindrical cavity. Lightweight DVAs and small HRs tuned to the natural frequencies of the targeted structural and acoustic modes, respectively, result in significant acoustic and structural attenuation when the devices are optimally damped. Simulations show that significant interior noise reduction can only be achieved by adding damping to both structural and acoustic modes, which are resonant in the frequency bandwidth of interest. In order to be independent of the azimuth angle of the excitation and to avoid unwanted modal interactions, the devices are distributed evenly around the cylinder in rings. This treatment can only achieve good performance if the structure and the acoustic cavity are lightly damped. (C) 2002 Acoustical Society of America. [DOI: 10.1121/1.1514933]
\end{abstract}

PACS numbers: 43.40.Tm, 43.40.At, 43.40.Sk [ANN]

\section{INTRODUCTION}

Large cylindrical structures are common in the aerospace industry. The reduction of noise transmitted into such enclosures is particularly challenging due to the high excitation levels, the complex nature of the disturbance, and the severe mass and volume constraints imposed on the design of the treatments. The development of lightweight structures, made of composite materials, has lowered the acoustic transmission loss of such structures and therefore further increased the acoustic transmission problem.

The transmission of sound into an enclosed acoustic cavity can be simplified into four stages: (i) coupling between the external noise and the structure, (ii) structural vibration, (iii) coupling between the structural vibration and the interior fluid, and (iv) the sound in the interior. Control treatments can operate at some or all of these stages in order to achieve attenuation of the interior noise levels.

Control devices can be attached to the structure in order to directly reduce the vibration level [stage (ii)] and in order to reduce the structural acoustic coupling [stages (i) and (iii)]. For example, Huang and Fuller ${ }^{1,2}$ used dynamic absorbers to reduce the interior sound level in an aircraft fuselage, at a single excitation frequency, by reducing the structural velocity. Guigou et al. ${ }^{3}$ extended this work by detuning the absorbers in order to reduce the coupling between the structure and the interior sound field. For broadband applications it becomes difficult to reduce the structural acoustic coupling using lightweight treatment as decoupling at one frequency tends to increase coupling at another. Gardonio et al. $^{4}$ had some success achieving this using blocking

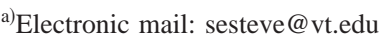

masses placed on the structure but had to assume some knowledge of the angle of the incident noise field. Jolly and Sun $^{5}$ used vibration absorbers to reduce the radiation of broadband noise from a vibrating panel and Nagaya and $\mathrm{Li}^{6}$ examined the optimization of an absorbers treatment applied to a radiating plate using neural network but neither considered radiation into a cavity.

The other approach to control the sound transmission is to directly treat the sound in the enclosure [stage (iv)]. Absorptive materials, such as acoustic blankets, perform well in the high-frequency range, but are unsuitable for lowfrequency control due to the volume and mass constraints imposed in aerospace applications. However, acoustic attenuation can be obtained in the low-frequency range by the use of Helmholtz resonators (HR). Fahy and Schofield ${ }^{7}$ investigated the interaction between a single optimally damped HR and an acoustic mode in an enclosure and Cummings ${ }^{8}$ extended the analysis to a resonator array and its effect on the sound field in a cavity. Also, Doria ${ }^{9}$ tried to broaden the functional frequency range of a HR by using a resonator with multiple natural frequencies. In all these studies, the acoustic disturbance is generated by an arbitrary source distribution inside the cavity and is not excited by a structure.

The contribution of this work lies in the simultaneous application of both structural and acoustic control devices to a fully coupled structural-acoustic system [stages (ii) and (iv)]. Multiple optimally damped distributed vibration absorbers (DVAs) and HRs are applied to control the sound transmission in a cylindrical enclosure over a broad frequency range containing many structural and acoustic resonances. A conventional modal expansion method ${ }^{4}$ is used to describe the behavior of the cylindrical shell, excited by an external acoustic plane wave, and its acoustic cavity. The 


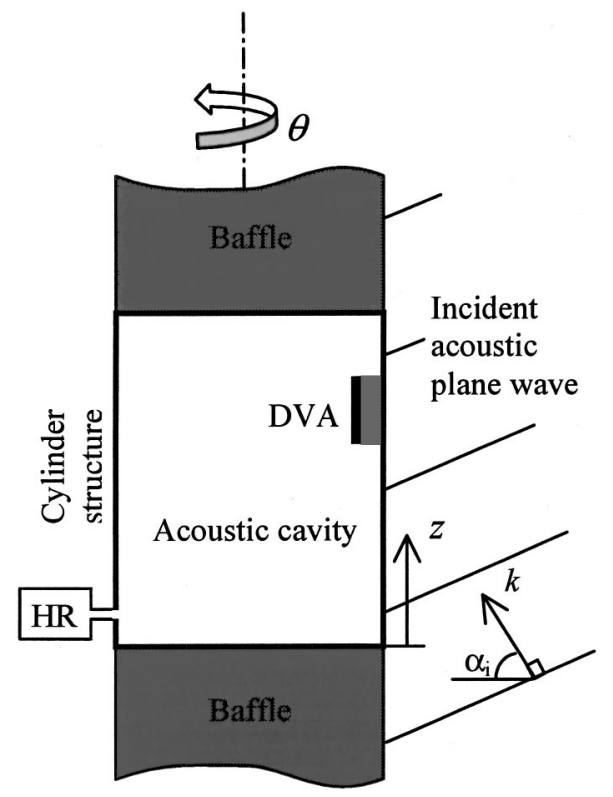

FIG. 1. Test cylinder mounted in an infinite baffle and excited by an external acoustic plane wave treated with HRs and DVAs.

optimization of the DVA treatment based on the studies by Johnson et al. ${ }^{10,11}$ is extended by analogy to the HR treatment. All the elements are then fully coupled using an impedance matching method to compute the interior acoustic attenuation provided by the noise reduction devices.

\section{THEORY}

In this section the analytical formulation of the problem is introduced. The system being modeled is shown in Fig. 1. It is constituted of a simply supported cylinder embedded in an infinite rigid baffle excited by an acoustic plane wave. To control the vibration of the cylinder, DVAs can be attached anywhere on its surface except the top and bottom disks, which are not excited by the exterior acoustic field due to the presence of the baffle. HRs can be placed anywhere inside the cylinder, but in order to maintain a simple model for the acoustic cavity they are assumed to lie outside and to couple to the enclosed fluid at the circumference of the structure. The behavior of the structure and the acoustic cavity is described using a modal approach. The system is put in a matrix form, and using an impedance matching method is then fully coupled with the noise reduction devices. Once the necessary components of the model are defined, the expression for the vibration and interior acoustic response of the system is derived.

\section{A. Cylinder structural model}

The dynamic behavior of thin cylindrical shells has generated a multitude of theory based on different assumptions and approximations. The comparison of these different theories has also been subject to valuable studies, such as the work done by Leissa. ${ }^{12}$ For the purpose of this work, the cylinder is assumed to be thin, isotropic, and made out of a homogeneous material whose mechanical properties are adjusted in order to match the behavior of an experimental composite prototype. Therefore within the framework of this study, the standard Donnell-Mushtari theory, even though inaccurate for the low circumferential wave number, gives a sufficient first approximation of the resonant frequencies. The shape of the $\left(n_{s}, m_{s}\right)$ structural mode shapes $\Psi_{n_{s} m_{s}}^{s}$ (the "s" superscript or subscript signifies that the variable refers to the structure) is given by

$$
\Psi_{n_{s} m_{s}}^{s}(z, \theta)=\frac{1}{\sqrt{\Lambda_{n_{s} m_{s}}}} \sin \left(\frac{\pi n_{s} z}{L}\right)\left\{\begin{array}{c}
\cos \left(m_{s} \theta\right) \\
\sin \left(m_{s} \theta\right)
\end{array}\right\},
$$

where the mode orders are $1 \leqslant n_{s} \leqslant n_{\max }, 0 \leqslant m_{s} \leqslant m_{\max }, L$ is the length of the cylinder, $\Lambda_{n_{s} m_{s}}$ is the normalization factor of the $\left(n_{s}, m_{s}\right)$ mode, such that $\iint_{S}\left(\Psi_{n_{s} m_{s}}^{s}\right)^{2} d S=S$, and $S$ denotes the surface of the cylinder. Because of their symmetry, cylinders have circumferential modes whose orientation depends on the excitation location. Therefore, the cylinder is considered to have two orthogonal circumferential modes of the same order $m_{s}$ : one sine and one cosine. These two modes are independent and although they have the same resonant frequency, the incident acoustic field excites them differently. This is equivalent to a single mode with an orientation angle that changes depending on the excitation. The out-of-plane velocity $w$ can be described as a modal summation by

$$
w(\theta, z, \omega)=\sum_{N_{s}} v_{n_{s} m_{s}}(\omega) \Psi_{n_{s} m_{s}}^{s}(\theta, z)
$$

where $v_{n_{s} m_{s}}$ is the complex mode amplitude, and $N_{s}$ is the total number of structural modes considered. Once the structural natural frequency $\omega_{n_{s} m_{s}}$ is obtained, the modal mobility of the cylinder $A_{n_{s} m_{s}}^{s}$ is derived using the second-order system equation:

$$
A_{n_{s} m_{s}}^{s}(\omega)=\frac{i \omega}{M\left(\omega_{n_{s} m_{s}}^{2}-\omega^{2}+2 \xi_{n_{s} m_{s}} i \omega_{n_{s} m_{s}} \omega\right)},
$$

where $M$ is the mass of the cylinder, $\xi_{n_{s} m_{s}}$ the modal damping ratio, and $A_{n_{s} m_{s}}^{s}$ denotes a modal velocity over a modal force. In the simulations, the modal damping ratios are adjusted to be representative of the observable damping level in real composite cylinders. In the case of structures vibrating in dense fluid such as submarines, the effect of fluid loading (or radiation loading) on the structure dynamics must be taken into account. In the present case, the external radiation loading can be neglected because of the low density of air. The complex amplitude $v_{n_{s} m_{s}}$ of each structural mode is obtained by multiplying the mobility of the cylinder $A_{n_{s} m_{s}}^{s}$ by the total modal force $F_{n_{s} m_{s}}$ applied to the cylinder. Writing this in a matrix form yields

$$
\mathbf{v}=\mathbf{A}^{\mathrm{s}} \mathbf{F}
$$

where $\mathbf{A}^{\mathbf{s}}$ is an $N_{s} \times N_{s}$ diagonal matrix of modal mobilities obtained with Eq. (3), $\mathbf{F}$ is an $N_{s} \times 1$ vector of modal forces, and $\mathbf{v}$ is an $N_{s} \times 1$ vector of structural modal velocities.

Part of the force exerted on the cylinder is due to the incident acoustic plane wave. In order to calculate the external acoustic pressure acting on a cylinder, it is necessary to account for the scattering caused by the cylinder. The exter- 
nal pressure at the cylinder surface $P_{\text {ext }}$ is a function of the azimuth and elevation angles $\theta_{i}$ and $\alpha_{i}$, respectively, and of the frequency $\omega$ of the wave, as shown in Fig. 1. Since the cylindrical baffle is assumed to be infinite, the scattering is only a function of angle $\theta$. The simple phase dependence in the $z$ direction can then be calculated from the axial wave number in air $k_{z}=k \sin \alpha_{i}$, where $k=\omega / c$, and $c$ is the speed of sound in the fluid. From Morse and Ingard,${ }^{13}$ the pressure around the cylinder expressed in terms of cylindrical waves is the sum of the incident field pressure $P_{i}$ and the scattered field pressure $P_{s}$ :

$P_{\text {ext }}\left(\theta, z, \omega, \theta_{i}, \alpha_{i}\right)=P_{i}\left(\theta, z, \omega, \theta_{i}, \alpha_{i}\right)+P_{s}\left(\theta, z, \omega, \theta_{i}, \alpha_{i}\right)$.

The amplitude of each scattered cylindrical wave is derived using a hard wall boundary condition. The pressure distribution $P_{\text {ext }}$ on the surface of the cylinder due to an incident plane wave of amplitude $P_{0}$ is thus expressed as a summation of cylindrical waves of circumferential amplitude $P_{m},{ }^{13}$

$$
\begin{aligned}
& P_{\text {ext }}\left(\theta, z, \omega, \theta_{i}, \alpha_{i}\right) \\
& \quad=P_{0} e^{i\left(k_{z} z-\omega t\right)} \sum_{m=0}^{\infty} P_{m}\left(\omega, \alpha_{i}\right) \cos \left(m\left(\theta-\theta_{i}\right)\right) .
\end{aligned}
$$

The exterior modal force $F_{n_{s} m_{s}}^{\mathrm{ext}}$ that excites each mode is thus obtained by integrating the external pressure over the structural mode shape:

$$
F_{n_{s} m_{s}}^{\mathrm{ext}}\left(\omega, \alpha_{i}, \theta_{i}\right)=\iint_{S} \Psi_{n_{s} m_{s}}^{s} P_{\mathrm{ext}}\left(\theta, z, \omega, \alpha_{i}, \theta_{i}\right) d S,
$$

where the structural mode shapes $\Psi_{n_{s} m_{s}}^{s}$ are given in Eq. (1). Note that any kind of force can be decomposed on the structural modal base using this method.

\section{B. Structure-acoustic cavity coupling}

In this section, the elements of the acoustic cavity model are derived using a similar approach as for the previously derived structural model, after which the coupling mechanisms between the two models are presented.

\section{Cylinder acoustic model}

Using Bessel functions ${ }^{13} J_{m}$, the acoustic mode shapes $\Psi^{a}$ for a circular cylindrical enclosure of radius $R$ are given by

$\Psi_{n m p}^{a}(r, \theta, z)=\frac{1}{\sqrt{\Lambda_{n m p}}} J_{m}\left(k_{m p} r\right)\left\{\begin{array}{c}\cos (m \theta) \\ \sin (m \theta)\end{array}\right\} \cos \left(\frac{\pi n z}{L}\right)$,

where $\Lambda_{n m p}$ is the normalization factor such that $\iiint_{V}\left(\Psi_{n m p}^{a}\right)^{2} d V=V, V$ is the volume of the cavity, and the superscript " $a$ " signifies that the variable refers to the acoustic cavity. The circumferential wave numbers $k_{m p}$ are derived from the hard wall boundary condition $\left.(\partial / \partial r) J_{m}\left(k_{m p} r\right)\right|_{r=R}$ $=0$. The resonant frequency of the $(n, m, p)$ mode is thus given by $\omega_{n m p}=\sqrt{k_{n}^{2}+k_{m p}^{2}}$, where $k_{n}=n \pi / L$ is the axial modal wave number. As with the structural modes, the cir- cumferential orientation of an acoustic mode results from the combination of two independent orthogonal modes, one sine and one cosine, of the same order $m$. At any point $(r, \theta, z)$ inside the cylinder, the acoustic pressure $p(r, \theta, z, \omega)$ is approximated by the modal summation:

$$
p(r, \theta, z, \omega)=\sum_{\mathrm{N}_{\mathrm{a}}} p_{n m p}(\omega) \Psi_{n m p}^{a}(r, \theta, z),
$$

where $p_{n m p}$ is the complex mode amplitude, and $\mathrm{N}_{\mathrm{a}}$ the total number of acoustic modes considered. The acoustic modal impedance of the enclosed fluid $A_{n m p}^{a}$ defined as a modal pressure over a modal acoustic source strength is given by

$$
A_{n m p}^{a}(\omega)=\frac{\rho c^{2} i \omega}{V\left(\omega_{n m p}^{2}-\omega^{2}+2 i \xi_{n m p} \omega_{n m p} \omega\right)},
$$

where $\rho$ is the air density and $\xi_{n m p}$ the modal damping ratio. This damping is incorporated to account for the absorption of the acoustic treatment that is usually present in real applications but is typically very small at low frequencies. Once an $\mathrm{N}_{\mathrm{a}} \times 1$ vector of acoustic modal source strengths $\mathbf{u}$ is defined, the $\mathrm{N}_{\mathrm{a}} \times 1$ vector of acoustic modal pressures $\mathbf{p}$ can be expressed by

$$
\mathbf{p}=\mathbf{A}^{\mathrm{a}} \mathbf{u}
$$

where $A^{a}$ is the $N_{a} \times N_{a}$ diagonal matrix of modal acoustic impedances of the cylinder calculated from Eq. (10). Once the components of the structural and acoustic model are defined, the two models are coupled together, as described below.

\section{Structural-acoustic spatial coupling}

The coupling coefficient $C$ between a structural and an acoustic mode is computed by integration of the product of their shapes over the cylinder surface at $\mathrm{r}=\mathrm{R}$,

$$
C=\int_{0}^{L} \int_{0}^{2 \pi} \Psi_{n_{s} m_{s}}^{s}(\theta, z) \Psi_{n m p}^{a}(R, \theta, z) R d \theta d z .
$$

Due to the orthogonality of the sine and cosine function, the structural cosine circumferential mode couples only with the cosine circumferential acoustic modes and likewise with the sine modes. The coupling coefficients have the dimension of a surface $\left(\mathrm{m}^{2}\right)$. These coefficients can thus form an $\mathrm{N}_{\mathrm{a}} \times \mathrm{N}_{\mathrm{s}}$ coupling matrix $\mathbf{C}$, whose elements are the result of the integral in Eq. (12),

$$
\begin{aligned}
C\left([n, m, p],\left[n_{s}, m_{s}\right]\right) \\
\quad=\frac{2 R L J_{m}\left(R k_{m p}\right)}{\epsilon_{m} \sqrt{\Lambda_{n_{s} m_{s}} \Lambda_{n m p}}} \frac{n_{s}\left|(-1)^{n}-(-1)^{n_{s}}\right|}{\left(n_{s}^{2}-n^{2}\right)} \delta_{m, m_{s}},
\end{aligned}
$$

where the Kronecker delta symbol $\delta_{m, m_{s}}$ is zero if $m \neq m_{s}$ and unity if $m=m_{s}$ and the Neumann symbol $\boldsymbol{\epsilon}_{m}$ equals 1 if $m=0$ and equals 2 if $m \neq 0$. Therefore, to obtain a coupling coefficient different than zero, the circumferential orders $m_{s}$ and $m$ of the structural and acoustic mode must be equal and the axial orders $n_{s}$ and $n$, must define an odd-even or evenodd combination. Due to the $\left(n_{s}^{2}-n^{2}\right)$ term in the denomi- 


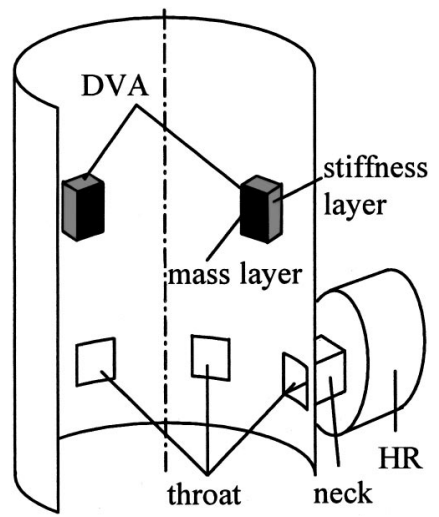

FIG. 2. Coupling between the cylinder and the noise reduction devices: HRs and DVAs.

nator of Eq. (13), modes with greatly different axial mode orders will be poorly coupled. As shown by Gardonio et al., ${ }^{4}$ the properties of the coupling coefficients $C$ determine the number of modes required in the simulations. The number of acoustic modes can be reduced to those whose resonant frequencies lie inside a band slightly larger than the one of interest, however, the structural modes that are well coupled to the acoustic modes must be included, even though their resonant frequencies lie well outside the band of interest.

The matrix $\mathbf{C}$ represents the link between the structural and acoustic model. By means of its physical dimension $\left(\mathrm{m}^{2}\right)$, it converts the $\mathrm{N}_{\mathrm{s}} \times 1$ structural modal velocity vector $\mathbf{v}$ into an $\mathrm{N}_{\mathrm{a}} \times 1$ modal acoustic source strength vector $\mathbf{u}$, or, reciprocally, it converts the $\mathrm{N}_{\mathrm{a}} \times 1$ modal acoustic pressure vector $\mathbf{p}$ into an $\mathrm{N}_{\mathrm{s}} \times 1$ internal modal force vector $\mathbf{F}^{\text {int }}$ :

$$
\begin{aligned}
& \mathbf{u}=\mathbf{C v}, \\
& \mathbf{F}^{\mathrm{int}}=-\mathbf{C}^{\mathrm{T}} \mathbf{p} ;
\end{aligned}
$$

$\mathbf{C}^{\mathbf{T}}$ denotes the transpose of $\mathbf{C}$ and the minus sign used in Eq. (15) yields a positive $\mathbf{F}^{\text {int }}$ in the inward normal direction.

\section{Coupling of the noise reduction devices}

In this section we present the modeling of the noise reduction devices and their coupling to the structural and acoustic model.

\section{DVA and HR modeling}

As shown in Fig. 2, a DVA consists of a distributed spring typically made of acoustic polyurethane foam on which is placed a distributed mass. Therefore, a DVA can be considered as a vibration absorber acting over a surface area $s_{d}$. The reaction force of the DVA induced by the velocity of the structure represents the DVA impedance $Z_{d}$ :

$$
Z_{d}(\omega)=m_{d} \frac{i \omega \omega_{d}^{2}+2 \zeta_{d} \omega^{2} \omega_{d}}{\left(\omega_{d}^{2}-\omega^{2}\right)-2 i \zeta_{d} \omega \omega_{d}}
$$

where $\omega_{d}$ is the natural frequency, $m_{d}$ the mass, and $\zeta_{d}$ the damping ratio of the DVA. The terms $Z_{d}(\omega)$ are grouped into an $N_{d} \times N_{d}$ diagonal DVA impedance matrix $\mathbf{Z}_{d}$, where $N_{d}$ is the number of DVAs.
A HR consists of a rigid wall cavity of volume $V_{h}$ and a neck of cross section area $s_{h}$ and length $l_{h}$, as shown in Fig. 2. If all its dimensions are small compared to the acoustic wavelength, a HR can be modeled as an acoustic equivalent to a mechanical vibration absorber, where the air in the cavity acts like a spring, and the air in the neck like a lumped mass. The interior radiation mass effect is included by a correction factor ${ }^{14}$ added to $l_{h}$, yielding an equivalent neck length $l_{e}$. The external radiation loading is accounted for by the summation of the $\mathrm{N}_{\mathrm{a}}$ acoustic modes at the HR throat. Since this is a near-field effect, the convergence of the velocity amplitude at the throat of the HR with increasing $\mathrm{N}_{\mathrm{a}}$ was checked. At resonance, the HR throat velocity converges quickly as only a $0.16 \mathrm{~dB}$ magnitude difference between $\mathrm{N}_{\mathrm{a}}$ $=76$ and $\mathrm{N}_{\mathrm{a}}=273$ occurs. Thus, 76 is set as a lower bound for $\mathrm{N}_{\mathrm{a}}$. Using a second-order spring-mass system equation, the HR acoustic admittance, expressed as a volume velocity over a pressure, is given by

$$
Y_{h}(\omega)=\frac{s_{h} i \omega}{\rho l_{e}\left[\left(\omega_{h}^{2}-\omega^{2}\right)-2 i \zeta_{h} \omega \omega_{h}\right]},
$$

where $\omega_{h}=c \sqrt{s_{h} / V_{h} l_{e}}$ is the HR resonant frequency, and $l_{e}$ $=l_{h}+0.85 \sqrt{s_{h} / \pi}$ for a square necked resonator. The admittance terms $Y_{h}(\omega)$ are grouped in an $\mathrm{N}_{\mathrm{h}} \times \mathrm{N}_{\mathrm{h}}$ diagonal HR admittance matrix $\mathbf{Y}_{\mathbf{h}}$, where $\mathrm{N}_{\mathrm{h}}$ is the number of HR.

In order to couple DVAs and HRs to the structural and acoustic model described in the previous sections, the velocity and pressure inputs to the impedance and admittance of the DVA and HR, respectively, are expressed using a modal summation at the location of the devices. This location on the surface of the cylinder with respect to a particular mode shape (node or antinode) defines a level of spatial coupling between the device and the different modes, as shown in the next section.

\section{Structure-DVA and acoustic-HR spatial coupling}

The coupling between a DVA and the cylinder is obtained by integrating each structural mode shape over the DVA rectangular surface of attachment $s_{d}=b \times a$ at its desired location $\left(\theta_{0}, z_{0}\right)$ on the cylinder. The contact surface between the cylinder and the DVA is assumed to be flat. Normalized by $s_{d}$, the dimensionless coupling coefficients $\varphi_{n_{s} m_{s}}^{s}$, are given by

$\varphi_{n_{s} m_{s}}^{s}\left(\theta_{0}, z_{0}, s_{d}\right)=\frac{1}{s_{d}} \int_{z_{0}-b / 2}^{z_{0}+b / 2} \int_{\theta_{0}-\Delta \theta}^{\theta_{0}+\Delta \theta} \Psi_{n_{s} m_{s}}^{s}(\theta, z) R d \theta d z$,

where $\Delta \theta=\sin ^{-1}(a / 2 R)$. The structural modes in the mass layer of the DVA itself are not taken into account; it is assumed that the DVA applies a uniformly distributed normal force on the cylinder. The coefficients $\varphi_{n_{s} m_{s}}^{s}$ form $\boldsymbol{\Phi}^{\mathrm{s}}$, a fully populated $\mathrm{N}_{\mathrm{d}} \times \mathrm{N}_{\mathrm{s}}$ matrix that couples $\mathrm{N}_{\mathrm{d}}$ DVAs to $\mathrm{N}_{\mathrm{s}}$ structural modes.

In a similar manner, the coupling between a HR and the enclosed fluid is computed by integrating each acoustic mode shape over the square area $\left(s_{h}=a \times a\right)$ of the resonator throat at its desired location $\left(r_{0}, \theta_{0}, z_{0}\right)$ in the cylinder. In the simulations the resonators are assumed to lie outside the cyl- 
inder and to couple at the circumference $\left(r_{0}=R\right)$. This is considered to be comparable to placing HRs inside the cavity, as long as the HR dimensions are small compared to the acoustic wavelength and have a small volume. By making this assumption, the mode shapes remain unchanged, and this simplifies the simulation considerably. As with the DVAs coupling, the HR throat is assumed flat over $s_{h}$,

$\varphi_{n m p}^{a}\left(\theta_{0}, z_{0}, s_{h}\right)=\frac{1}{s_{h}} \int_{z_{0}-a / 2}^{z_{0}+a / 2} \int_{\theta_{0}-\Delta \theta}^{\theta_{0}+\Delta \theta} \Psi_{n m p}^{a}(R, \theta, z) R d \theta d z$.

The coefficients $\varphi_{n m p}^{a}$ form a fully populated $\mathrm{N}_{\mathrm{h}} \times \mathrm{N}_{\mathrm{a}}$ matrix, $\boldsymbol{\Phi}^{\mathrm{a}}$, coupling $\mathrm{N}_{\mathrm{h}}$ HRs to $\mathrm{N}_{\mathrm{a}}$ acoustic modes. Using the different elements previously defined, the velocity and acoustic response of the fully coupled system is derived in the next section.

\section{Matrix formulation of the coupled system}

The coupling between all the components of the system is achieved using an impedance matching method. Therefore, the modal force $\mathbf{F}^{\text {DVA }}$ exerted on the structure by the DVAs is expressed as a function of the matrix $\boldsymbol{\Phi}^{\mathbf{s}}$, its transpose $\boldsymbol{\Phi}^{\mathbf{s} \mathbf{T}}$, the diagonal matrix $\mathbf{Z}_{\mathbf{d}}$, and the structural modal vibration vector $\mathbf{v}$ :

$$
\mathbf{F}^{\mathrm{DVA}}=\boldsymbol{\Phi}^{\mathrm{sT}} \mathbf{Z}_{\mathbf{d}} \boldsymbol{\Phi}^{\mathrm{s}} \mathbf{v}
$$

Assuming the velocity distribution in the throat of the HR to be uniform over the surface $s_{h}$, HRs act as acoustic piston sources. Therefore, the total acoustic modal source strength of the coupled system $\mathbf{u}$ is the sum of two quantities, $\mathbf{u}^{\mathbf{h}}$ and $\mathbf{u}^{\mathbf{s}}$. The modal source strength produced by the HR $\mathbf{u}^{\mathbf{h}}$ is a function of the acoustic modal pressure $\mathbf{p}$, and the acoustic modal source strength due to the structure $\mathbf{u}^{\mathrm{s}}$ given by Eq. (14) is a function of $\mathbf{v}$,

$$
\mathbf{u}=\mathbf{u}^{\mathbf{h}}+\mathbf{u}^{\mathrm{s}}=\boldsymbol{\Phi}^{\mathrm{aT}} \mathbf{Y}_{\mathbf{h}} \boldsymbol{\Phi}^{\mathrm{a}} \mathbf{p}+\mathbf{C v}
$$

Using Eq. (11), the acoustic modal pressure vector $\mathbf{p}$ due to the total acoustic source strength $\mathbf{u}$ becomes

$$
\mathbf{p}=\mathbf{A}^{\mathrm{a}}\left(\boldsymbol{\Phi}^{\mathrm{aT}} \mathbf{Y}_{\mathbf{h}} \boldsymbol{\Phi}^{\mathrm{a}} \mathbf{p}+\mathbf{C v}\right) .
$$

The total force $\mathbf{F}$ exciting the cylinder is the sum of the external acoustic force $\mathbf{F}^{\text {ext }}$, the internal acoustic force $\mathbf{F}^{\text {int }}$ given by Eq. (15), and the reacting force of DVAs $\mathbf{F}^{\text {DVA }}$ given by Eq. (20). Expanding the vector $\mathbf{F}$ in Eq. (4) into these three components, the structural modal velocity vector $\mathbf{v}$ becomes

$$
\mathbf{v}=\mathbf{A}^{\mathrm{s}}\left(\mathbf{F}^{\mathrm{ext}}-\mathbf{C}^{\mathbf{T}} \mathbf{p}+\boldsymbol{\Phi}^{\mathrm{sT}} \mathbf{Z}_{d} \Phi^{\mathrm{s}} \mathbf{v}\right) .
$$

Equations (22) and (23) are two coupled matrix equations defining the behavior of the fully coupled system. Solving this system of two equations yields $\mathbf{v}$ and $\mathbf{p}$ as a function of the external acoustic modal force $\mathbf{F}^{\text {ext }}$,

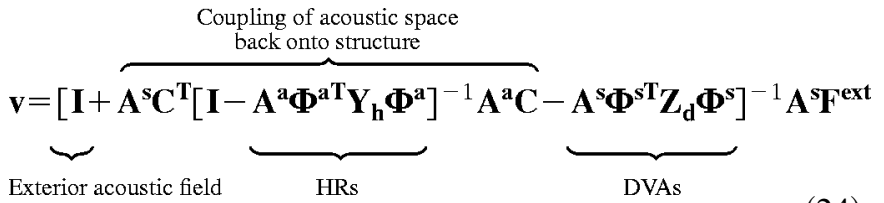

Assuming the interior acoustic space to be relatively uncoupled from the structure, and so neglecting the internal acoustic force $\mathbf{F}^{\text {int }}$ in comparison to the external acoustic force $\mathbf{F}^{\mathbf{e x t}}$, the expression for $\mathbf{v}$ and $\mathbf{p}$ can be simplified to

$$
\begin{aligned}
& \mathbf{v}=[\mathbf{I}-\overbrace{\mathbf{A}^{\mathbf{s}} \boldsymbol{\Phi}^{\mathbf{s} \mathbf{T}} \mathbf{Z}_{\mathbf{d}} \boldsymbol{\Phi}^{\mathbf{s}}}^{\text {DVAs }}]^{-1} \mathbf{A}^{\mathbf{S}} \mathbf{F}^{\mathbf{e x t}} \\
& \mathbf{p}=[\mathbf{I}-\underbrace{\mathbf{A}^{\mathrm{a}} \boldsymbol{\Phi}^{\mathrm{a} \mathbf{T}} \mathbf{Y}_{\mathbf{h}} \boldsymbol{\Phi}^{\mathrm{a}}}_{\text {HRs }}]^{-1} \mathbf{A}^{\mathrm{a}}\left[\mathbf{C}[\mathbf{I}-\underbrace{\mathbf{A}^{\mathrm{s}} \boldsymbol{\Phi}^{\mathbf{s} \mathbf{T}} \mathbf{Z}_{\mathbf{d}} \boldsymbol{\Phi}^{\mathrm{s}}}_{\text {DVAs }}]^{-1} \mathbf{A}^{\mathrm{S}} \mathbf{F}^{\mathbf{e x t}}\right] .
\end{aligned}
$$

Several simulations using different damping ratios for the structure and the acoustic cavity, with different configurations of DVA and HR, have shown that the difference in the obtained noise reduction using Eqs. (26), (27) instead of Eqs. (24), (25), respectively, are negligible.

As is shown in the simplified equations (26) and (27), the vibration of the cylinder is only affected by the DVAs; however, the internal acoustic field represented by $\mathbf{p}$ is modi- fied by both HRs and DVAs. In order to obtain an average sound pressure level independent of the location inside the cylinder, the total time average acoustic potential energy $E_{p}$ is computed as

$$
E_{p}(\omega)=\frac{1}{4 c^{2} \rho} \iint_{V} \int|p(\omega, r, \theta, z)|^{2} d V
$$

If the modal expression for the pressure given by Eq. (9) is substituted into Eq. (28), the orthonormal properties of the modes allow the acoustic potential energy ${ }^{15}$ to be computed using $\mathbf{p}$ and its Hermitian transpose $\mathbf{p}^{\mathbf{H}}$.

$$
E_{p}\left(\omega, \alpha_{i}, \theta_{i}\right)=\frac{V}{4 \rho c^{2}} \sum_{N_{s}}\left|p_{n m p}\left(\omega, \alpha_{i}, \theta_{i}\right)\right|^{2}=\frac{V}{4 \rho c^{2}} \mathbf{p}^{\mathbf{H}} \mathbf{p} .
$$

Similarly, the total structural kinetic energy is used as an indicator of the average vibration level of the cylindrical 
TABLE I. Geometry and physical properties used in the numerical simulations.

\begin{tabular}{ll}
\hline \hline Density of air $\rho$ & $1.19 \mathrm{~kg} \mathrm{~m}^{-3}$ \\
Speed of sound in air $c$ & $343 \mathrm{~ms}^{-1}$ \\
Density of structure $\rho_{s}$ & $380 \mathrm{~kg} \mathrm{~m}^{-3}$ \\
Young's Modulus $E$ & $3.5 \mathrm{e} \mathrm{Pa}$ \\
Length of cylinder $L$ & $2.81 \mathrm{~m}$ \\
Radius of cylinder $R$ & $1.23 \mathrm{~m}$ \\
Thickness of cylinder & $0.01 \mathrm{~m}$ \\
\hline
\end{tabular}

structure. Due to the orthogonality and normalization of the structural modes, the total structural kinetic energy $E_{k}$ can be expressed by summing the square of the $\mathrm{N}_{\mathrm{s}}$ structural modal velocities, which is equal to the product of $\mathbf{v}$ by its Hermitian transpose $\mathbf{v}^{\mathbf{H}}$,

$E_{k}\left(\omega, \alpha_{i}, \theta_{i}\right)=\frac{1}{2} M \sum_{N_{s}}\left[v_{n_{s} m_{s}}\left(\omega, \alpha_{i}, \theta_{i}\right)\right]^{2}=\frac{1}{2} M \mathbf{v}^{\mathbf{H}} \mathbf{v}$.

\section{NUMERICAL SIMULATION}

The previously developed analytical formulation is applied to a cylinder whose geometry and physical properties are summarized in Table I. The incident acoustic wave excites the structure with an elevation angle $\alpha_{i}=70^{\circ}$ and an azimuth angle $\theta_{i}=0^{\circ}$. The structural and acoustic cavity damping ratios are set to $1 \%$ in order to be representative of the damping levels encountered below $200 \mathrm{~Hz}$ in a typical composite cylindrical enclosure.

\section{A. Bare cylinder response to incident acoustic field}

In this section we present the main sound transmission mechanisms in agreement with the detailed study by Gardonio et ll. $^{4}$ The coupling between the external field and the structural modes characterizes the excitation of the cylinder. This external acoustic-structural coupling is represented by the external modal force $F_{n_{s} m_{s}}^{\text {ext }}$, which, after integration of Eq. (7), reduces to

$$
\begin{aligned}
F_{n_{s} m_{s}}^{\mathrm{ext}}\left(\omega, \alpha_{i}\right)= & P_{0} \frac{2 R \pi e^{-i \omega t}}{\sqrt{\Lambda_{n_{s} m_{s}}}} \frac{n_{s} \pi / L}{k_{z}^{2}-\left(n_{s} \pi / L\right)^{2}} \\
& \times\left[(-1)^{n_{s}} e^{i k_{z} L}-1\right] P_{m_{s}}\left(\omega, \alpha_{i}\right) .
\end{aligned}
$$

The circumferential amplitude $P_{m_{s}}$ denotes the scattering of the wave by the cylinder, and is plotted in Fig. 3(a) for different $m_{s}$ as a function of frequency. This amplitude $P_{m_{s}}$, behaves like a "high-pass filter" whose cut-on frequency increases with the circumferential mode order $m_{s}$, except for the breathing mode $m_{s}=0$, which has a maximum value at 0 Hz. The axial component represented by the term $\left\{n_{s} \pi / L /\left[k_{z}^{2}-\left(n_{s} \pi / L\right)^{2}\right]\right\}\left[(-1)^{n_{s}} e^{i k_{z} L}-1\right]$ is the Fourier wave number transform of the axial mode shape $\sin \left(n_{s} \pi / L\right)$. It represents the spatial coupling between the axial wave number in air $k_{z}=k \sin \alpha_{i}$ and the axial modal wave number $k_{n_{s}}=n_{s} \pi / L$ in the cylinder. This coupling is therefore characterized by a main lobe near the coincidence frequency between $k_{z}$ and $k_{n_{s}}$ and sidelobes of decaying amplitude. However, for $n_{s}=1$, the axial modal wave number $k_{n_{s}}$ represents

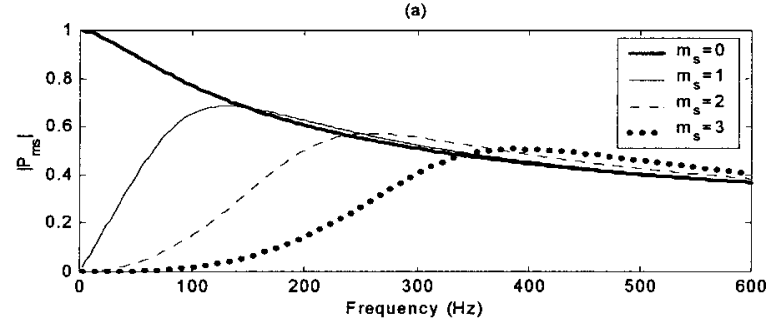

(b)

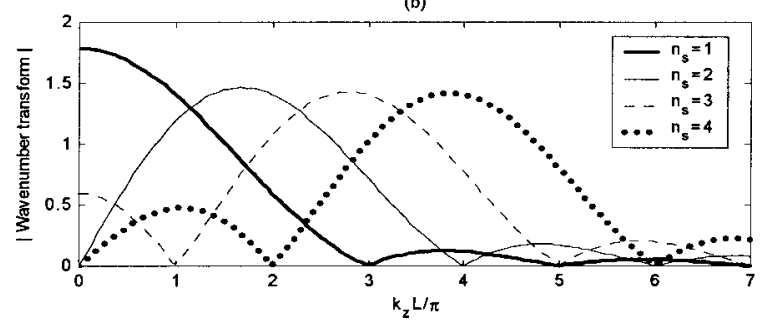

FIG. 3. (a) Magnitude of the circumferential amplitude $P_{m_{s}}$ of $F_{n_{s} m_{s}}^{\text {ext }}$ due to an incident plane wave of $1 \mathrm{~Pa}$. $\left(\alpha_{i}=70^{\circ}, \theta_{i}=0^{\circ}\right)$ as a function of the excitation frequency for $m_{s}=0,1,2,3$. (b) Magnitude of the Fourier wave number transform of $\sin \left(n_{s} \pi / L\right)$ as a function of the normalized axial wave number in air for $n_{s}=1,2,3,4$.

only half of a wave along the length of the cylinder and so has its main lobe at $k_{z}=0$. This axial component of $F_{n_{s} m_{s}}^{\text {ext }}$ is plotted in Fig. 3(b) as a function of the normalized axial wave number in air. Although 36 structural modes with circumferential order as high as 13 resonate below $200 \mathrm{~Hz}$, Fig. 3 shows that only modes with circumferential order $m_{s} \leqslant 3$ and axial order $n_{s} \leqslant 5$ are well excited by the incident field. This is confirmed by Fig. 4, which shows that the structural kinetic energy of the bare cylinder, computed using all the modes resonating in the band, is dominated by only three lower-order modes. This filtering effect allows a reduction in the number of structural modes necessary to obtain convergence of the simulated results.

The internal acoustic response, plotted on top of the structural kinetic energy in Fig. 4 is composed of both acoustic resonances and structural resonances well excited by the incident acoustic field. The level of coupling between a structural and an acoustic mode is both spatial and frequency related. In Fig. 4, the $(1,2)$ structural mode is well coupled to the $(0,2,0)$ acoustic mode due to a maximum of the spatial

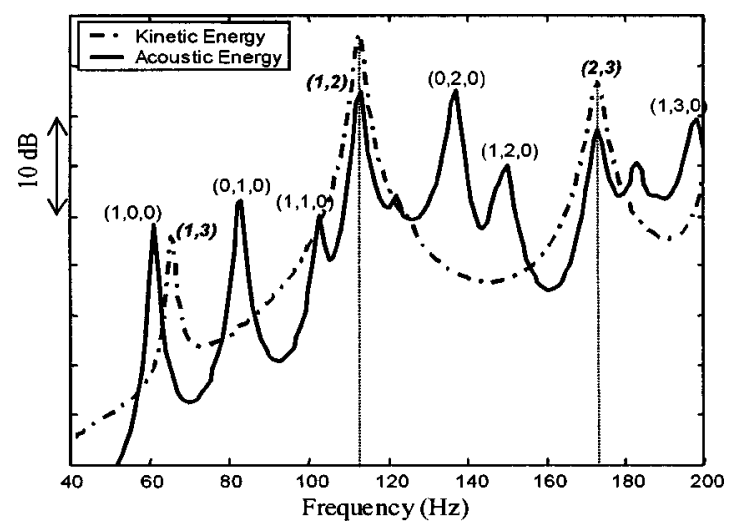

FIG. 4. Overlay of the structural kinetic and total acoustic potential energy curves due to an incident plane wave $\left(\alpha_{i}=70^{\circ}, \theta_{i}=0^{\circ}\right)$, with structural mode order (bold italic) and acoustic mode order. 
coupling coefficient $C([0,2,0],[1,2])$ given by Eq. (13), and also due to the proximity of the two resonances, $112 \mathrm{~Hz}$ for the $(1,2)$ mode, and $137 \mathrm{~Hz}$ for the $(0,2,0)$ mode. Therefore, the $(0,2,0)$ acoustic mode is responsible for nearly all of the response in the 100-150 Hz range. Similarly, in the 160-200 $\mathrm{Hz}$ range, the coupling between the $(2,3)$ structural and the $(1,3,0)$ acoustic modes is responsible for the majority of the interior noise.

\section{B. Noise reduction mechanisms}

In this section we present the HRs' and DVAs' effects on the system and the strategies developed to optimize the noise attenuation. The mechanism of both HRs and DVAs is based on the dynamic vibration absorber system. ${ }^{16}$ Consider a vibration absorber of mass $m_{d}$ and natural frequency $\omega_{d}$ attached to a mass-spring system with mass $m$ and natural frequency $\omega_{n}$. Tuning the vibration absorber such that $\omega_{d}$ $=\omega_{n}$ splits the resonance of the system into two new resonances of similar amplitude on either side of $\omega_{n}$. The bigger the mass ratio, the farther apart the two resonances of the coupled system appear. By adding damping to the absorber (i.e., between the system and the absorber mass), both "new" resonances are well damped and significant broadband attenuation can be achieved. Depending on the type of excitation, several formulas for damping ratios ${ }^{16,17}$ lead to optimal vibration reduction. For a wide band random excitation, the optimal damping ratio $\zeta_{d}^{\text {opt }}$ derived by Korenev and Reznikov ${ }^{16}$ is expressed as

$$
\zeta_{d}^{\mathrm{opt}}=\sqrt{\frac{\nu(1+0.75 \nu)}{4(1+\nu)^{3}}}
$$

where $\nu=m_{d} / m$. Once coupled to a continuous structure of mass $M$, the effective mass ratio $\nu$ is weighted by the normalized mode shape squared at the absorber location $\left(\theta_{0}, z_{0}\right)$,

$$
\nu \simeq\left[\Psi_{n_{s} m_{s}}^{s}\left(\theta_{0}, z_{0}\right)\right]^{2} \frac{m_{d}}{M} .
$$

The coupling between a HR of volume $V_{h}$ and an acoustic mode of an enclosure of volume $V$ obeys the same mechanisms. By analogy with den Hartog's optimized dynamic absorber, ${ }^{17}$ Fahy and Schofield ${ }^{7}$ derive an optimal HR damping level $\zeta_{h}^{\text {opt }}$ as a solution of

$$
\mu^{2}\left(\frac{1}{2 \zeta_{h}^{o p t}}\right)^{4}+\frac{4 \mu}{\xi_{n m p}}\left(\frac{1}{2 \zeta_{h}^{\mathrm{opt}}}\right)^{3}+\mu\left(\frac{1}{2 \zeta_{h}^{\mathrm{opt}}}\right)^{2}-1=0,
$$

where $\xi_{n m p}$ is the damping of the enclosure and $\mu$ the effective volume ratio, given by

$$
\mu \simeq\left[\Psi_{n m p}^{a}\left(r_{0}, \theta_{0}, z_{0}\right)\right]^{2} \frac{V_{h}}{V} .
$$

In reality, the damping of the resonator is created by viscous losses of the air moving in the neck. Therefore the damping can be adjusted by placing small amounts of porous material in the HR throat. In a DVA, the damping is produced by structural losses in the acoustic foam as it compresses. Using different types of foam leads to different levels of damping for the DVAs. In both cases, the amount of vibration attenu-
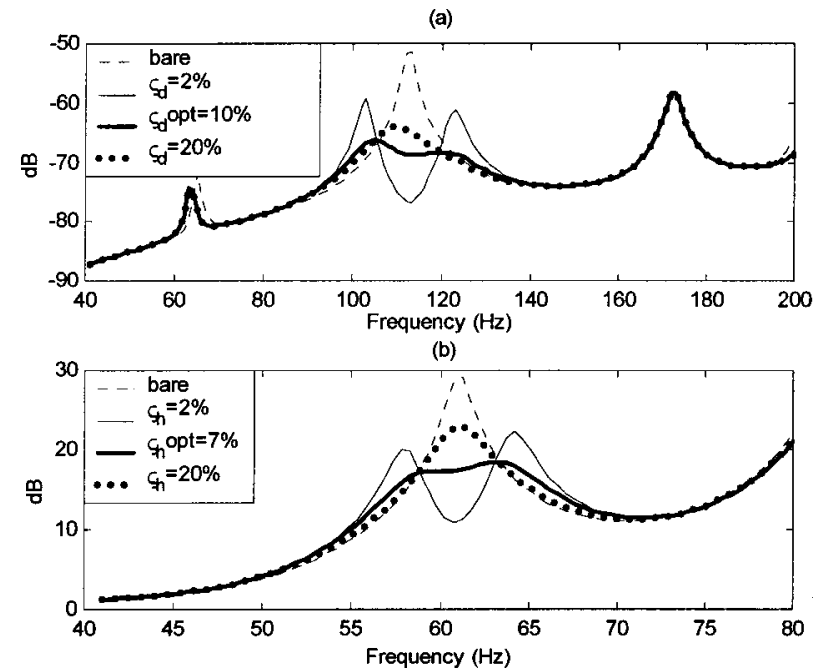

FIG. 5. (a) Influence of the damping ratio of the DVAs, $\zeta_{d}$, on the structural kinetic energy of the cylinder, the optimal damping ratio $\zeta_{d}^{\text {opt }}$ is given by Eq. (32). (b) Influence of the damping ratio of the HRs, $\zeta_{h}$, on the acoustic potential energy of the cylinder; the optimal damping ratio $\zeta_{h}^{\text {opt }}$ is given by Eq. (34).

ation is a weak function of the damping ratio, and thus a small variation about the optimal level only marginally degrades the performance of the HRs and DVAs.

To illustrate the mechanisms of the noise reduction devices, Fig. 5 shows the effect of the DVA and HR damping ratio on the structural kinetic energy (a) and the acoustic potential energy (b) response of the cylinder, respectively. With low damping, the two "new" modes are both fairly lightly damped and only small broadband noise reduction is achieved. Alternatively, if the damping is too high, the devices become uncoupled from the structural/acoustic mode and no longer dissipate energy.

In both cases the devices are split into several identical units distributed evenly around the circumference and tuned to the resonant frequency of the targeted mode: $112 \mathrm{~Hz}$ for the 13 DVAs and $61 \mathrm{~Hz}$ for the $5 \mathrm{HRs}$. Multiple devices are used for two reasons. First, using a symmetric ring of absorbers allows the treatment to be independent of the azimuth angle $\theta_{i}$ of the incident field that is assumed to be unknown. Consequently, only the axial mode shape component is used in the computation of the effective mass or volume ratio, which is weighed by $1 / \epsilon_{m}$ since half the mass or volume of the devices is effectively acting on circumferential modes different than zero. Second, the devices act as discontinuities that can couple modes together by shifting energy from one circumferential mode to another. For instance, a

TABLE II. Amplitude of the $(1,2)$ and $(3,8)$ mode [normalized by the $(1,2)$ mode amplitude of the bare cylinder] at $112 \mathrm{~Hz}$ for a 3 DVAs and 13 DVAs ring treatment tuned to $112.5 \mathrm{~Hz}$.

\begin{tabular}{cccc}
\hline \hline & \multicolumn{3}{c}{ Normalized mode amplitude at } \\
& Bare & $\begin{array}{c}112 \mathrm{~Hz}(\mathrm{~dB}) \\
\text { Ring of }\end{array}$ & Ring of 13 \\
Mode order & DVAs \\
(frequency) & cylinder & 3 DVAs & DV.5 \\
\hline $1,2(112.5 \mathrm{~Hz})$ & 0 & -5.5 & -18.5 \\
$3,8(112.7 \mathrm{~Hz})$ & -131 & -8.5 & -112 \\
\hline \hline
\end{tabular}


TABLE III. Acoustic modes below $160 \mathrm{~Hz}$.

\begin{tabular}{cc}
\hline \hline Mode order $(n, m, p)$ & Resonant frequency $(\mathrm{Hz})$ \\
\hline$(1,0,0)$ & 61 \\
$(0,1,0)$ & 82.4 \\
$(1,1,0)$ & 102.5 \\
$(2,0,0)$ & 122 \\
$(0,2,0)$ & 136.6 \\
$(2,1,0)$ & 147 \\
$(1,2,0)$ & 149.6 \\
\hline \hline
\end{tabular}

ring of $N_{d}$ DVAs targeting a mode of circumferential order $m_{s}=i$ redistributes the energy to all $m_{s}=\left|i \pm q N_{d}\right|$ modes, where $q$ is an integer. Thus, a DVA treatment is likely to excite structural modes that are not forced by the incident acoustic field. The closer the resonant frequency of the $m_{s}$ $=\left|i \pm q N_{d}\right|$ mode is to the DVAs' tuning frequency, the higher the excitation. Therefore, a large number of DVAs per ring ensures a weak modal coupling since only modes with greatly different circumferential order, which in most cases implies greatly different resonant frequencies, can interact. As an example, Table II presents the performance on the targeted $(1,2)$ structural mode of two different treatments, both weighing $2 \%$ of the total mass of the cylinder. A ring of 13 DVAs leads to an attenuation of $18 \mathrm{~dB}$ at the resonance, whereas a ring of 3 DVAs only reduces it by $5 \mathrm{~dB}$ and increases the amplitude of the $(3,8)$ mode, which is barely excited by the external acoustic field.

\section{Control of the $50-160 \mathrm{~Hz}$ band with DVAs and HRs}

In this section we present an example of a treatment designed to control the interior acoustic level from 50 to 160 Hz. In this frequency band, the enclosure presents only seven acoustic modes listed in Table III. The first three are well separated and so are targeted individually by three independent rings of $\mathrm{HR}$, whereas targeting two out of the last four modes ensures good reduction. Because the highest circumferential order below $230 \mathrm{~Hz}$ is $m=3$, five HRs per ring is sufficient to obtain a negligible excitation of higher-order modes. A ring of 13 DVAs tuned to $112 \mathrm{~Hz}$ is used to target the structural $(1,2)$ mode. Each ring of devices is placed on

TABLE IV. Noise reduction device characteristics for the treatment used in Fig. 6.

\begin{tabular}{|c|c|c|c|c|c|c|}
\hline \multicolumn{7}{|c|}{ Ring of 13 DVAs total mass $=1.6 \mathrm{Kg}$} \\
\hline $\begin{array}{l}\text { Targeted } \\
\text { mode }\end{array}$ & $\begin{array}{l}\text { Tuning } \\
\text { frequency } \\
(\mathrm{Hz})\end{array}$ & $\begin{array}{c}\text { Mass/M } \\
(\%)\end{array}$ & $\begin{array}{c}\nu \\
(\%)\end{array}$ & $\begin{array}{c}s_{d} \\
\left(\mathrm{~cm}^{2}\right)\end{array}$ & $\begin{array}{l}\zeta_{d}^{\mathrm{opt}} \\
(\%)\end{array}$ & $\begin{array}{c}z \\
(\mathrm{~m})\end{array}$ \\
\hline$(1,2)$ & 112.5 & 2 & 5 & 200 & 10 & 1.49 \\
\hline \multicolumn{7}{|c|}{ Rings of $5 \mathrm{Hrs}$ total volume $=0.8 \mathrm{~m}^{3}$} \\
\hline $\begin{array}{l}\text { Targeted } \\
\text { mode }\end{array}$ & $\begin{array}{l}\text { Tuning } \\
\text { frequency } \\
(\mathrm{Hz})\end{array}$ & $\begin{array}{c}\text { Volume/V } \\
(\%)\end{array}$ & $\begin{array}{c}\mu \\
(\%)\end{array}$ & $\begin{array}{c}s_{h} \\
\left(\mathrm{~cm}^{2}\right)\end{array}$ & $\begin{array}{l}\zeta_{h}^{\mathrm{opt}} \\
(\%)\end{array}$ & $\begin{array}{c}z \\
(\mathrm{~m})\end{array}$ \\
\hline$(1,0,0)$ & 61 & 0.5 & 1 & 22 & 6.7 & 0.02 \\
\hline$(0,1,0)$ & 82.4 & 0.5 & 2 & 22 & 9.6 & 1.43 \\
\hline$(1,1,0)$ & 102.5 & 1 & 8.3 & 223 & 18.7 & 0.07 \\
\hline$(0,2,0)$ & 136.6 & 2 & 14.8 & 354 & 24.6 & 1.5 \\
\hline$(1,2,0)$ & 149.6 & 2 & 14.7 & 346 & 24.6 & 0.07 \\
\hline
\end{tabular}

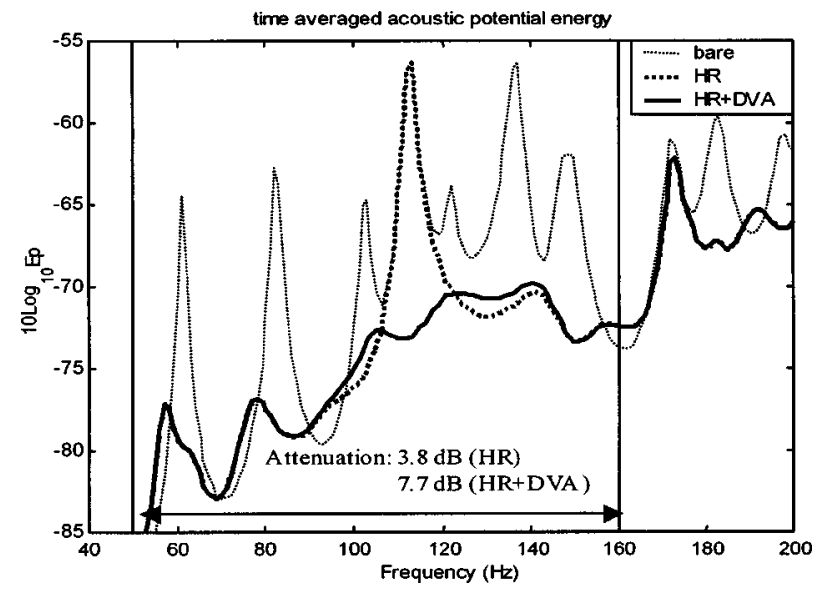

FIG. 6. Acoustic potential energy inside the cylinder excited by 1 Pa. Plane wave before and after treatment.

an axial antinode of the targeted mode shape to maximize the effective volume ratio $\mu$ or mass ratio $\nu$. The total volume of the HRs represents $6 \%$ of the cavity volume $V$, and the total mass of the DVAs $2 \%$ of the cylinder mass. The characteristics of this treatment are detailed in Table IV. Using sets of 76 acoustic modes and 36 structural modes, including the two orthogonal modes of the same circumferential order, the effect of the HRs and DVAs on the acoustic potential energy is plotted in Fig. 6. Although the optimally damped HRs reduce the acoustic resonances by more than $10 \mathrm{~dB}$, almost half of the energy transmitted in the $50-160 \mathrm{~Hz}$ band is due to the $(1,2)$ structural resonance, as shown in Fig. 6. The DVA treatment is therefore necessary to improve the noise reduction. Once the damping, the frequency, and the location of the devices are optimized, the performance of a treatment can only be improved by increasing both the total mass of DVAs and the total volume of HRs, as shown in Table V. Note that for each case, the damping is optimized with the new mass and volume ratios.

As explained previously, the DVA and HR noise reduction mechanism is based on adding damping to sharp structural and acoustic resonances responsible for the majority of the interior noise. Therefore, such treatment can be adapted to a different type of excitation by targeting in each different case the unfavorable resonances. However, the performance of these devices is directly related to the amount of damping initially present in the structure and in the acoustic cavity.

TABLE V. Attenuation in the $50-160 \mathrm{~Hz}$ band of the acoustic potential energy using the treatment described in Table IV for a different total mass of DVAs and total volume of HRs with optimal damping ratios computed accordingly.

\begin{tabular}{cccccc}
\hline \hline $\begin{array}{c}50-160 \mathrm{~Hz} \\
\text { attenuation }(\mathrm{dB})\end{array}$ & & \multicolumn{4}{c}{$\begin{array}{c}\text { Total volume of HRs } \\
(\% \text { of cavity volume })\end{array}$} \\
\cline { 1 - 3 } \cline { 4 - 6 } $\begin{array}{c}\text { Total mass of DVA } \\
\text { (\% of cylinder mass })\end{array}$ & & $3 \%$ & $6 \%$ & $9 \%$ & $12 \%$ \\
\hline $1 \%$ & 6.2 & 7.2 & 7.8 & 8.6 \\
$2 \%$ & 6.7 & 7.7 & 8.3 & 9.1 \\
$4 \%$ & 7.3 & 8.3 & 8.8 & 9.6 \\
\hline \hline
\end{tabular}


TABLE VI. HR-DVA treatment performance with different initial acoustic and structural damping ratios.

\begin{tabular}{cc}
\hline \hline $\begin{array}{c}\text { Acoustic and structural } \\
\text { damping ratios }(\%)\end{array}$ & $\begin{array}{c}\text { Noise attenuation } \\
\text { from } 50-160 \mathrm{~Hz}(\mathrm{~dB})\end{array}$ \\
\hline 0.5 & 9.9 \\
1 & 7.7 \\
3 & 4.4 \\
5 & 3.1 \\
10 & 1.6 \\
15 & 1.0 \\
\hline \hline
\end{tabular}

The higher the damping ratios of the system, the less reduction the treatment can provide. This is illustrated by Table VI, which displays the noise attenuation provided by the treatment described in Table IV over the 50-160 Hz band for different structural and acoustic initial damping ratios of the cylinder. When the structure and the acoustic cavity are lightly damped, i.e., structural and acoustic damping ratios below 3\%, the DVAs and HRs can provide significant noise attenuation.

The performance of a combined HRs/DVAs treatment is also robust with respect to the elevation angle of the external acoustic plane wave. As an example, simulations show that the treatment detailed in Table IV, which is designed for an elevation angle of $70^{\circ}$, achieves attenuation between 6.2 and $8.2 \mathrm{~dB}$ in the $50-160 \mathrm{~Hz}$ band for different elevation angles between $30^{\circ}$ and $80^{\circ}$. This is to be expected since the main mechanism of the control is damping and not modal restructuring, which is more sensitive to the primary excitation.

\section{CONCLUSIONS}

This work evaluated the ability of a combined DVA/HR treatment to reduce the sound transmission in an enclosed cylindrical shell excited by an external plane wave. Using a modal expansion and an impedance matching method, the structure, the interior acoustic field, and the noise reduction devices were fully coupled, leading to the analytical formulation of the structural kinetic energy and the acoustic potential energy of a treated cylindrical cavity.

The analysis shows that at low frequencies, the structural vibration is only dominated by a few lower-order modes because of the coupling between the plane wave and the cylinder. The favorable coupling between these modes and the cavity generates an acoustic response composed of both acoustic and structural resonances. As a consequence, a significant reduction of the interior acoustic levels can only be achieved by using DVAs and HRs simultaneously.

As an example, an overall reduction of $7.7 \mathrm{~dB}$ in the $50-160 \mathrm{~Hz}$ band is obtained by using DVAs weighing only $2 \%$ of the cylinder mass and HRs representing $6 \%$ of its volume. This result was obtained by tuning the devices to the natural frequency of the targeted modes and by using optimal damping ratios for both DVAs and HRs. The devices were used in rings to avoid unfavorable modal interactions and to obtain a treatment independent of the azimuth angle of excitation. Such treatment is also robust to variations in the elevation angle of excitation as it is based on adding damping to sharp structural and acoustic resonances, and not on reducing the structural-acoustic coupling. In conclusion, this work has shown that lightweight DVA and small HR treatment can significantly reduce the sound transmission in an enclosure as long as the structure and the cavity are lightly damped, which is usually the case at low frequency in aerospace applications.

\section{ACKNOWLEDGMENTS}

We acknowledge Vibro-Acoustic Sciences and Fuller Technologies for supporting part of this work.

${ }^{1}$ Y. M. Huang and C. R. Fuller, "The effects of dynamic absorbers on the forced vibration of a cylindrical shell and its coupled interior sound field," J. Sound Vib. 200, 401-418 (1997).

${ }^{2}$ Y. M. Huang and C. R. Fuller, "Vibration and noise control of the fuselage via dynamic absorbers," J. Vibr. Acoust. 120, 496-502 (1998).

${ }^{3}$ C. Guigou, J. P. Maillard, and C. R. Fuller, "Study of globally detuned absorbers for controlling aircraft interior noise," 4th International Congress on Sound and Vibration, St. Petersburg, Russia, June 1996.

${ }^{4}$ P. Gardonio, N. S. Fergusson, and F. J. Fahy, "A modal expansion analysis of the noise transmission through circular cylindrical shell structure with blocking masses," J. Sound Vib. 244, 259-297 (2001).

${ }^{5}$ M. R. Jolly and J. Q. Sun, "Passive tuned vibration absorbers for sound radiation reduction from vibrating panels," J. Sound Vib. 191, 577-583 (1996).

${ }^{6} \mathrm{~K}$. Nagaya and L. Li, "Control of sound noise radiated from a plate using dynamic absorbers under the optimization by neural network," J. Sound Vib. 208, 289-298 (1997).

${ }^{7}$ F. J. Fahy and C. Schofield, "Note on the interaction between a Helmholtz resonator and an acoustic mode of an enclosure," J. Sound Vib. 72, 365378 (1980).

${ }^{8}$ A. Cummings, "The effect of a resonator array on the sound field in a cavity," J. Sound Vib. 154, 25-44 (1992).

${ }^{9}$ A. Doria, "Control of acoustic vibrations of an enclosure by means of multiple resonators," J. Sound Vib. 181, 673-685 (1995).

${ }^{10}$ M. E. Johnson, C. R. Fuller, and P. Marcotte, "Optimization of distributed vibration absorber for sound transmission into a composite cylinder," Proceedings of the 7th AIAA/CEAS Aeroacoustics Conference, May 2001, p. 2232

${ }^{11}$ H. Osman, M. E. Johnson, C. R. Fuller, and P. Marcotte, "Interior noise reduction of composite cylinders using distributed vibration absorbers," in Ref. 10, p. 2230

${ }^{12}$ A. W. Leissa, "Vibration of shells," NASA-SP-288, 1973, Chap. 2, pp. $31-49$.

${ }^{13}$ M. Morse and K. U. Ingard, Theoretical Acoustics (McGraw-Hill, New York, 1986), Chap. 8, p. 400; Chap. 9, p. 511.

${ }^{14}$ L. E. Kinsler, A. R. Frey, A. B. Coppens, and J. V. Sanders, Fundamentals of Acoustics, 4th ed. (Wiley, New York, 2000), Chap. 10, pp. 284-286.

${ }^{15}$ P. A. Nelson and S. J. Elliott, Active Control of Sound (Academic, New York, 1992), Chap. 10, pp. 319-322.

${ }^{16}$ B. G. Korenev and L. M. Reznikov, Dynamic Vibration Absorbers (Wiley, New York, 1993), Chap. 1, p. 62.

${ }^{17}$ J. P. den Hartog, Mechanical Vibrations (Dover, New York, 1985), Chap. 3, pp. 93-106. 